\title{
Carácter de las prácticas pedagógicas de los estudiantes del ciclo complementario de la Normal Superior del Quindío en el proyecto escuela incluyente
}

\author{
Character of the students' pedagogíc practice in the complementary cycle at \\ Quindío's Normal Superior in the inclusive school project
}

\author{
Marleny Bulla De Torres*
}

Recibido: Febrero 20 de 2009

Aceptado: Octubre 1 de 2009

Correspondencia:Email:marbula@hotmail.com

\section{RESUMEN}

Las Escuelas Normales Superiores, instituciones acreditadas para brindar la formación inicial del docente de Preescolar y Básica Primaria, están en el deber de asumir el reto de la pertinencia y la calidad; en este sentido, la práctica pedagógica es la esencia que vivifica y promueve un estilo propio en el desempeño del normalista, implicándolo en su quehacer educativo.

La investigación: CARÁCTER DE LAS PRÁCTICAS PEDAGÓGICAS DE LOS ESTUDIANTES DEL CICLO COMPLEMENTARIO DE LA NORMAL SUPERIOR DEL QUINDIOO, EN EL PROYECTO ESCUELA INCLUYENTE, es un estudio cualitativo, de enfoque etnográficocrítico; radicó en hacer una lectura de las prácticas pedagógicas que realizan los/as normalistas superiores, en las comunidades donde se lleva a cabo el proceso de atención educativa a poblaciones en situación de vulnerabilidad social; para develar lo que significa la experiencia y precisar el perfil pedagógico de los estudiantes en sus prácticas, y al mismo tiempo construir el sentido de ser maestro en las actuales circunstancias del devenir histórico, político y social de la región.

Indagó el proceso pedagógico de los maestros en formación inicial, con la infancia, la adolescencia, la juventud, la familia y la comunidad; beneficiarios potenciales del proyecto Escuela Incluyente; con el propósito de comprender las relaciones pedagógicas en los escenarios comunitarios, bajo el marco de la inclusión educativa y social, como una práctica emergente, a partir de las necesidades identificadas en estas poblaciones; atendidas por la Institución formadora de formadores en su programa de proyección social.

Palabras clave: Prácticas Pedagógicas, Inclusión educativa - Exclusión, Relaciones Pedagógicas, Maestro /a en Formación Inicial, Vulnerabilidad Social.

\begin{abstract}
The Escuelas Normales Superiores (Normal Superior Schools), accredited institutions to provide initial training of teachers of Preschool and Basic Elementary school, are on duty to accept a challenge of importance and quality; in this sense, the pedagogical practice is the essence that gives life and promotes a style in teachers' performance, generally involved in their educational task.

This research is a qualitative study, from an ethnographic-critical approach, which consisted on reviewing the pedagogic practices of students from the Normal Superior School in the communities in which the process of educational care for vulnerable populations in society is being developed; in order to discover what this experience actually means, and to refine the educational profile of students in their practices, while building their sense of being a teacher in the present historical, political and social circumstances of the region.

This project also inquired the learning process of teachers in initial training, with childhood, adolescence, youth, family and community, potential beneficiaries of the Inclusive School Project, with the aim of understanding the pedagogical relations in community settings, under the framework of educational and social inclusion, as an emerging practice, based on the needs identified in these populations served by the trainer institution in its social outreach program.

Keywords: Pedagogical Practices, Educational Inclusion - Exclusion, Educational Relations, Teacher in basic training, social
\end{abstract}

* Docente Escuela Normal Superior Del Quindío. 


\section{INTRODUCCIÓN}

as Escuelas Normales, a lo largo y ancho de la historia de Colombia se han ocupado de la formación de los maestros y maestras para las nuevas generaciones.

Actualmente, estas instituciones viven un proceso de reestructuración que inició con la Ley General de Educación 115, en el año 1994, y más específicamente a partir de la promulgación del decreto 3012 de 1997. Durante dicho proceso se han cerrado aproximadamente unas sesenta (60) Escuelas Normales en todo el país.

En el departamento del Quindío, en Armenia su capital, aún se conserva una de ellas, la cual recibió la Acreditación Previa en noviembre de 1999, y posteriormente, en julio del año 2003, obtuvo la Acreditación de Calidad y Desarrollo, encontrándose hoy día en su fase de auto evaluación e implementación del plan de mejoramiento, con el fin de fortalecer su propuesta de formación pedagógica bajo los presupuestos que sustenta la teoría crítica de la educación, y de esta manera propender por la verificación del cumplimiento de las condiciones básicas de calidad del programa deformación complementaria, a efectuarse en el año 2009.

Los/as estudiantes normalistas que desarrollen el perfil y deseen ser maestros, ingresan al programa de formación complementaria, el cual tiene un tiempo de duración de cuatro (4) semestres, correspondiente a los grados doce (12) y trece (13). Durante este periodo se profundiza en la formación pedagógica e investigativa y se consolida el proceso de las prácticas pedagógicas, perfilando de esta manera al nuevo /a maestro /a, quien al culminar la primera parte de sus estudios superiores, recibe el título de NORMALISTA SUPERIOR, otorgado por la Escuela Normal Superior del Quindío (en este caso).

La investigación se realizó dentro del marco de las Prácticas Pedagógicas de los maestros /as en formación inicial, desde la perspectiva de la Inclusión Educativa, o como en la actualidad lo asume el Ministerio de Educación Nacional, Educación Inclusiva con Calidad; específicamente, involucra el estudio y análisis de una experiencia particular que vive un grupo de estudiantes a partir de la Atención Educativa a Poblaciones Vulnerables del municipio de Armenia, dentro del Proyecto Pedagógico Institucional de Proyección Social denominado: ESCUELA INCLUYENTE. En la Escuela Normal Superior del Quindío, el proceso de educación comunitaria referido, surgió hace ocho (8) años, ofrecido a un grupo de comunidades socialmente vulnerables, víctimas de la exclusión educativa; en la actualidad se ha extendido a todas las zonas vulnerables de la ciudad de Armenia, donde generalmente la población, por múltiples razones no accede al servicio educativo.

Es un trabajo que parte de las necesidades educativas, promueve la escuela y se inserta en la familia y la comunidad, su filosofía privilegia la garantía de derechos de la niñez y la adolescencia, lo mismo que el empoderamiento familia comunidad y la corresponsabilidad social.

El proyecto de investigación se inscribe en el paradigma cualitativo, como un estudio etnográfico, crítico, que se propone indagar acerca de la realidad que viven estos estudiantes superiores (maestros /as en formación inicial) en el desarrollo de sus Prácticas Pedagógicas de enfoque social, lo cual les confiere un perfil particular e innovador, al igual que un sentido propio a la razón de ser maestros y maestras en las presentes épocas caracterizadas por la movilidad social y los conflictos de todo orden, que alteran el desarrollo y la promoción del ser humano.

Desde esta mirada, la investigación que a continuación se describe busca mejorar la calidad de la oferta educativa en la institución formadora de formadores, en pro de la formación de un nuevo docente, con destino a una sociedad cada vez más cambiante y polivalente que le impone retos y coloca en aprietos al sistema.

Se ha seleccionado como unidad de análisis el Campo de la Práctica Pedagógica de los estudiantes del Ciclo Complementario dentro del proceso de educación inclusiva y como unidad de trabajo quienes realizan sus prácticas con la población vulnerable objetivo del proyecto mencionado, no obstante, porque es en el escenario de la clase y en la cotidianeidad escolar donde se manifiestan permanentemente diversas pautas e interacciones socialmente establecidas, cuyos significados deben ser develados a fin de describir, interpretar, categorizar y comprender los fenómenos que rodean la enseñanza e identificar los procesos sociales que movilizan la realidad escolar, en ambientes de exclusión social.

La lectura vivencial de tales prácticas permitirá valorar su impacto y establecer su sentido frente a la formación inicial de los maestros y maestras para las complejidades sociales que tanto necesita la región y el país. 


\section{METOdOLOGÍA}

Criterio Investigativo El criterio metodológico que direccionó la investigación es el derivado de la etnografía, método centrado en la comparación constante, como un ejercicio de preguntarle a los datos de manera insistente; cuyo sentido interpretativo-aplicativo, conllevó a recrear las prácticas y comportamientos de los actores en el proceso de atención educativa a poblaciones infantiles y adolescentes vulnerables, en contextos de exclusión social, donde el hecho deviene en dato, el dato en mensaje y el mensaje en sentido, lo que finalmente, contribuyó a caracterizar el proceso de la práctica de los normalistas superiores desde la conjetura institucional.

Su análisis está sustentado a partir de la teoría fundamentada. Por lo cual, los procedimientos de interpretación conllevaron a la organización y clasificación de los datos, en conceptos y categorías, cada una en términos de sus propiedades y dimensiones.

Método: "Teoría Fundamentada": Corbin y Strauss. Se refiere a una teoría derivada de datos recopilados de manera sistemática y analizada por medio de un proceso de investigación. La característica primordial de este método es la fundamentación de conceptos en los datos, y la creatividad de los investigadores como un ingrediente esencial (pensamiento crítico y creativo).

El análisis está apoyado en la corriente interpretativa, desde cuyo eje se establecen la comprensión e interpretación de los fenómenos socioculturales anotados desde los significados de sus propios actores. En el desarrollo de la investigación, se utilizaron cuatro (4) instrumentos: a) la Observación Participante; b) el Diario de Campo; c) el Grupo Focal y d) La
Entrevista en Profundidad.

\section{Unidad de Análisis y de trabajo}

Se seleccionó como unidad de análisis el Campo de la Práctica Pedagógica de los estudiantes del Ciclo Complementario dentro del proceso de educación inclusiva y como unidad de trabajo quienes realizan sus prácticas con la población vulnerable objetivo del proyecto mencionado.

Dicha unidad de análisis se definió como el grupo de personas que conocen el proyecto Escuela Incluyente y que de alguna manera están o han estado involucrados en su desarrollo, son ellos/as quienes han vivido los procesos de búsqueda, encuentro, consolidación e intervención; a partir de su experiencia se viene pensando y generando una nueva propuesta de escuela como un lugar de acogida y de crecimiento personal que rompe con lo programático para instaurar un movimiento renovador garante de oportunidades educativas para las poblaciones socialmente vulnerables de la ciudad de Armenia Quindío.

La composición del muestreo teórico comprendió las siguientes categorías:

* Normalistas Superiores egresados que practicaron y fueron gestores del proyecto.

* Directivos de la Escuela Normal Superior del Quindío y maestros/as coordinadores de la práctica pedagógica, incluidos coordinadores de los colegios que acompañan el desarrollo de la propuesta (Nacional, ITI.).

* Estudiantes Normalistas del Ciclo Complementario. Practicantes actuales en el proyecto (primero y segundo año de formación docente).

* Comunidades. Familia, niñez, adolescencia y líderes comunitarios.

\begin{tabular}{|c|l|l|l|l|}
\hline Número & \multicolumn{1}{|c|}{ Escenario } & \multicolumn{1}{|c|}{ Caracterización } & \multicolumn{1}{c|}{$\begin{array}{c}\text { Método } \\
\text { Instrumento }\end{array}$} & \multicolumn{1}{c|}{ Objetivo } \\
\hline 7 & $\begin{array}{l}\text { Comunidades: } \\
\text { sentamiento temporal } \\
\text { San José, la Cecilia, } \\
\text { Bambusa. }\end{array}$ & $\begin{array}{l}\text { Gestores del proyecto } \\
\text { y coordinadores, } \\
\text { informantes clave. }\end{array}$ & $\begin{array}{l}\text { Observación: } \\
\text { Participante Grupo } \\
\text { focal. } \\
\text { Entrevista en } \\
\text { profundidad. }\end{array}$ & $\begin{array}{l}\text { Identificar los procesos } \\
\text { génesis y desarrollo del } \\
\text { proyecto, la proyección } \\
\text { que le dieron y lo que ha } \\
\text { significado para su vida } \\
\text { de maestros. }\end{array}$ \\
\hline 8 & $\begin{array}{l}\text { Comunidades: } \\
\text { Rojas Pinilla, la Cecilia, } \\
\text { Numbusa, }\end{array}$ & $\begin{array}{l}\text { Coordinadores } \\
\text { de cada sede, } \\
\text { Informantes clave. }\end{array}$ & $\begin{array}{l}\text { Observación: } \\
\text { Participante Grupo } \\
\text { focal. } \\
\text { Entrevista en } \\
\text { profundidad. }\end{array}$ & $\begin{array}{l}\text { Establecer el grado de } \\
\text { importancia del proyecto } \\
\text { dentro de su formación } \\
\text { superior. }\end{array}$ \\
\hline
\end{tabular}




\begin{tabular}{|c|c|c|c|c|}
\hline $\begin{array}{l}5 \text { familias, } \\
2 \text { Repre- } \\
\text { sentantes } \\
\text { de la } \\
\text { comunidad } \\
\text { (líderes } \\
\text { comunita- } \\
\text { rios. }\end{array}$ & $\begin{array}{l}\text { Sedes: } \\
\text { Rojas Pinilla, la Cecilia, } \\
\text { Bambusa, } \\
\text { Nuevo Berlín. }\end{array}$ & $\begin{array}{l}\text { Familias: } \\
\text { Que participaron en la } \\
\text { creación del proyecto, que } \\
\text { aún permanecen o son } \\
\text { nuevas en el proceso. } \\
\text { Líderes comunales: } \\
\text { Participante gestor. } \\
\text { Participante actual. }\end{array}$ & $\begin{array}{l}\text { Observación: } \\
\text { Participante Grupo focal. } \\
\text { Entrevista en } \\
\text { profundidad. }\end{array}$ & $\begin{array}{l}\text { Conocer la percepción que } \\
\text { tiene la comunidad acerca } \\
\text { del proyecto, lo que ha } \\
\text { significado en la educación } \\
\text { de la familia. }\end{array}$ \\
\hline
\end{tabular}

Figura 1. Matriz Analítica del Muestreo Teórico

\section{RESULTADOS Y ANÁLISIS DE LA INFORMACIÓN}

\section{Codificación Abierta}

Los conceptos fueron identificados a partir de la división de los contenidos en porciones o unidades temáticas que expresaban una idea central, dichas porciones se clasificaron con una expresión denominada categoría descriptiva, lo cual en este trabajo orientado desde el sentido e intencionalidad de develar lo que significan las prácticas pedagógicas de los normalistas superiores en el proyecto Escuela Incluyente, han emergido. Hasta aquí, la codificación abierta arrojó los siguientes resultados:

La Escuela Incluyente es una experiencia educativa que tiene como finalidad brindar oportunidades educativas a la infancia y adolescencia de comunidades socialmente vulnerables. Se desarrolla en la ciudad de Armenia Quindío, orientada desde la Escuela Normal Superior, directamente ejecutada por los estudiantes del ciclo complementario de formación docente, durante el transcurso de sus prácticas pedagógicas.

En este sentido, el estudio cualitativo del fenómeno que representa el carácter o el significado de las prácticas pedagógicas en procesos de inclusión a cargo de los estudiantes normalistas, nos muestra cómo la acción de educar en la Escuela Incluyente constituye en esencia la proyección social tanto de la escuela normal superior como del maestro en formación inicial, cuyas variables representativas entrelazan conceptos o categorías que se han codificado así:

1. Prácticas pedagógicas

2. Actores y escenarios

3. Vulnerabilidad social

4. Formación inicial del maestro

5. Proceso incluyente

6. Sentido y significado de ser maestro

7. Impacto y compromiso institucional y social.

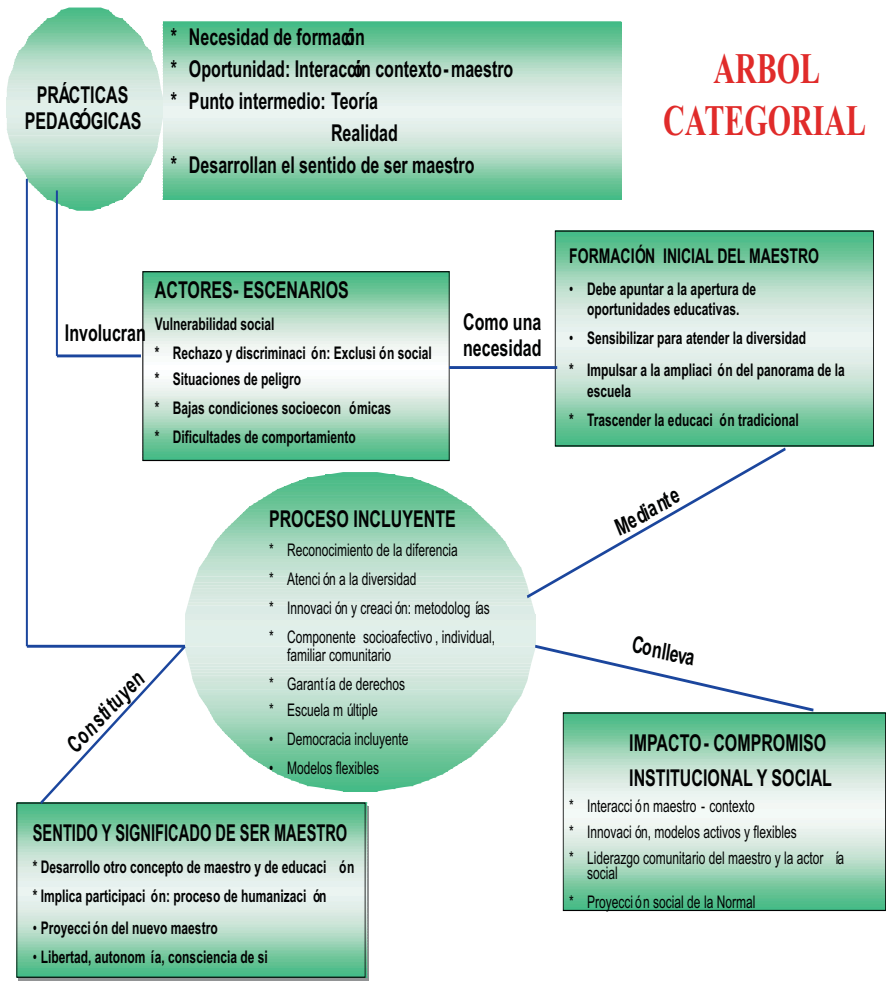

Figura 2. Árbol Categorial: describe las categorías emergentes del estudio

Las categorías identificadas se mueven en torno a la formación de los docentes para una nueva emergencia social que agencia grandes cambios a nivel no solo de la familia y de la escuela, sino también del Estado y de la sociedad.

De esta manera, las prácticas pedagógicas, se constituyen en la concreción del plan de estudios pensado y acordado para un grupo de población determinado, su ejecución se hace a través de la relación pedagógica, la cual toma diversos matices, de acuerdo con el docente asesor y la propuesta didáctica que este privilegie. Los equipos docentes requieren 
desarrollar esfuerzos coordinados para apoyar el proceso de enseñanza y aprendizaje, en la comunicación recíproca, las relaciones horizontales y la negociación con los estudiantes. Es así como en este escenario, se apropian la comunicación, la cogestión del aprendizaje y la relación afectiva, como elementos facilitadores del proceso de enseñanza y aprendizaje, por lo cual, su sentido y validación se evidencian en la organización del aula y en las estrategias de aprendizaje y de evaluación puestas en juego.

El experimentar una propuesta educativa que incluya a los más vulnerados socialmente, implica la creación de otros espacios y otras relaciones de tipo pedagógico, implica conocer y analizar las condiciones reales de las poblaciones objetivo de intervención, lo cual, en un momento dado pone en crisis la estructura enraizada del sistema educacional. En tal sentido, la institución formadora de formadores, ha de ponerse al día en materia de inclusión, avistando su mirada desde sus estudiantes, futuros egresados en su calidad de maestros y maestras de las nuevas generaciones, capacitados para ofertar modelos educativos alternativos y flexibles, que satisfagan sus necesidades de formación y desarrollo.

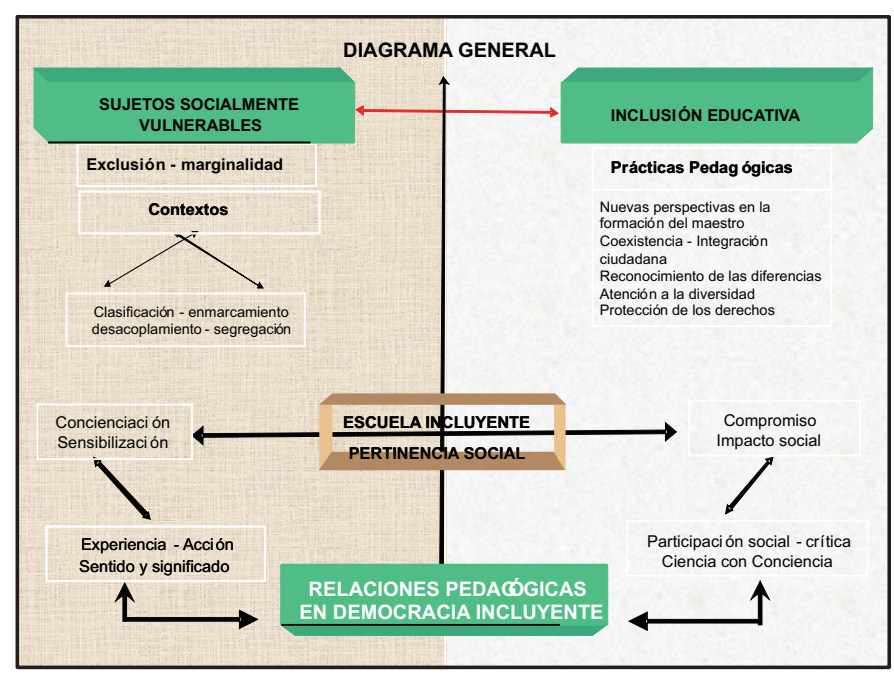

Figura 3. Desarrollo de la Categoría Central. Relaciones Pedagógicas en Democracia Incluyente

El propósito de indagar sobre el carácter de las prácticas pedagógicas, específicamente en la experiencia de inclusión educativa como elemento de formación de los nuevos maestros normalistas superiores, condujo a identificar una macro categoría denominada: RELACIONES PEDAGÓGICAS EN DEMOCRACIA INCLUYENTE, a partir de la cual cobran significado tanto la actuación de los maestros y las maestras en formación y sus formadores, como la proyección y el posicionamiento social de la Normal en la perspectiva de cambio y respuesta coherente a las necesidades yretos que nos plantean los sistemas sociales y culturales en este siglo. Valls (2004) afirma: "la educación es la clave de la inclusión y la convivencia social, pero para garantizar de verdad la igualdad de oportunidades, las escuelas deben transformarse hacia la apertura, el diálogo, la colaboración en red, la optimización del aprendizaje y la solidaridad" ( $p$. 58).

El análisis de dicha categoría central se orienta a establecer las coordenadas que a su interior legitimen y potencien la experiencia en la Escuela Incluyente, como un espacio educativo donde se brindan nuevas oportunidades para aquellos que hacen parte de las minorías, quienes conforman en la ciudad de Armenia una franja humana en situación de vulneración y vulnerabilidad social.

Como lo expresaba Paulo Freire, "las personas somos seres de transformación y no de adaptación, y solo así podemos plantear posibilidades de cambio educativo real y superar de esta forma la segregación y la desigualdad sociales"

El tema central del estudio se enfoca en la construcción de unas nuevas relaciones pedagógicas, para ser desplegadas y puestas en acción en un espacio de apertura, de reconversión de estructuras y procesos que han venido desdibujando la riqueza cultural, política y generacional de todos los tiempos. En tal sentido, sería plausible pensar, de acuerdo con Julio Rogero (2000) que:

La diversidad y la igualdad competen a la sociedad y a la escuela como parte de la sociedad, por ello hay que potenciar el afán transformador y la capacidad de transformación. En la sociedad red hay que incrementar la coordinación entre los centros educativos y la red de servicios y organizaciones sociales barriales y ciudadanas; es muy importante la pedagogía de la acción; transformar la información en conocimiento y el conocimiento en sabiduría. Todo ello en fraternidad, utopía que hará compatible la libertad con la igualdad (Julio Rogero. Conferencia- Educación: Igualdad + Diversidad. Año 2000. Valencia, España).

La interacción que establece el maestro con el grupo de estudiantes, es un pilar del proceso educativo. Los equipos pedagógicos en cada sector de la ciudad de Armenia, donde se lleva a cabo la inclusión de la población objetivo, han identificado diversas situaciones que les obliga a pensar en otras perspectivas y caminos a la hora de desarrollar la acción educativa.

Aunque el recorrido por el proyecto ha sido realizado por muchos practicantes, no siempre se ha podido detectar el mismo interés y compromiso en el que hacer pedagógico, sin embargo, los informantes de este estudio, dan a entender 
que para vincularse en las comunidades y ejercitar un buen papel, se requiere desarrollar ciertas competencias, las cuales hasta ahora no han sido imperativo en los espacios generales de la práctica, y por ello, algunos estudiantes practicantes, no alcanzan a superar las expectativas que nacen en la cotidianeidad de dicha experiencia.

Aprender a relacionarse en la Escuela Incluyente, es un sentir general, percibido por los actores informantes de la investigación; desarrollar nuevas ideas, buscar nuevas rutas para interaccionar y convivir en afecto, solidaridad y armonía con el entorno del barrio, la familia, los grupos de pares y las asociaciones vecinales, ofertando la enseñanza y el aprendizaje de los contenidos escolares, aparece como una necesidad trascendental, que al tenor de la investigación, ratifica y vigoriza la tesis central, bajo la cual se propone la emergencia de unas RELACIONES PEDAGÓGICAS EN DEMOCRACIA INCLUYENTE, que privilegien la inclusión del otro, enfocada en tres dispositivos que apuntan a la construcción de sujeto: necesidad y otredad-necesidad de sujeto - necesidad de conciencia. De tal manera que a partir del encargo social heredado a la Escuela Normal Superior del Quindío, se contribuya efectivamente al desarrollo y movilización de prácticas pedagógicas, encarnadas en la formación para la potenciación de sujeto.

La categoría central del estudio, converge en la construcción y desarrollo de unas relaciones pedagógicas que permeen los procesos de la educación en democracia incluyente, donde unos sujetos vulnerables, expuestos a la marginalidad y la exclusión, acceden a la educación formal, a través de una práctica pedagógica que promueve nuevas perspectivas en la formación del maestro, enfocadas en la coexistencia, la integración ciudadana, el reconocimiento de las diferencias, la atención a la diversidad y la protección de los derechos.

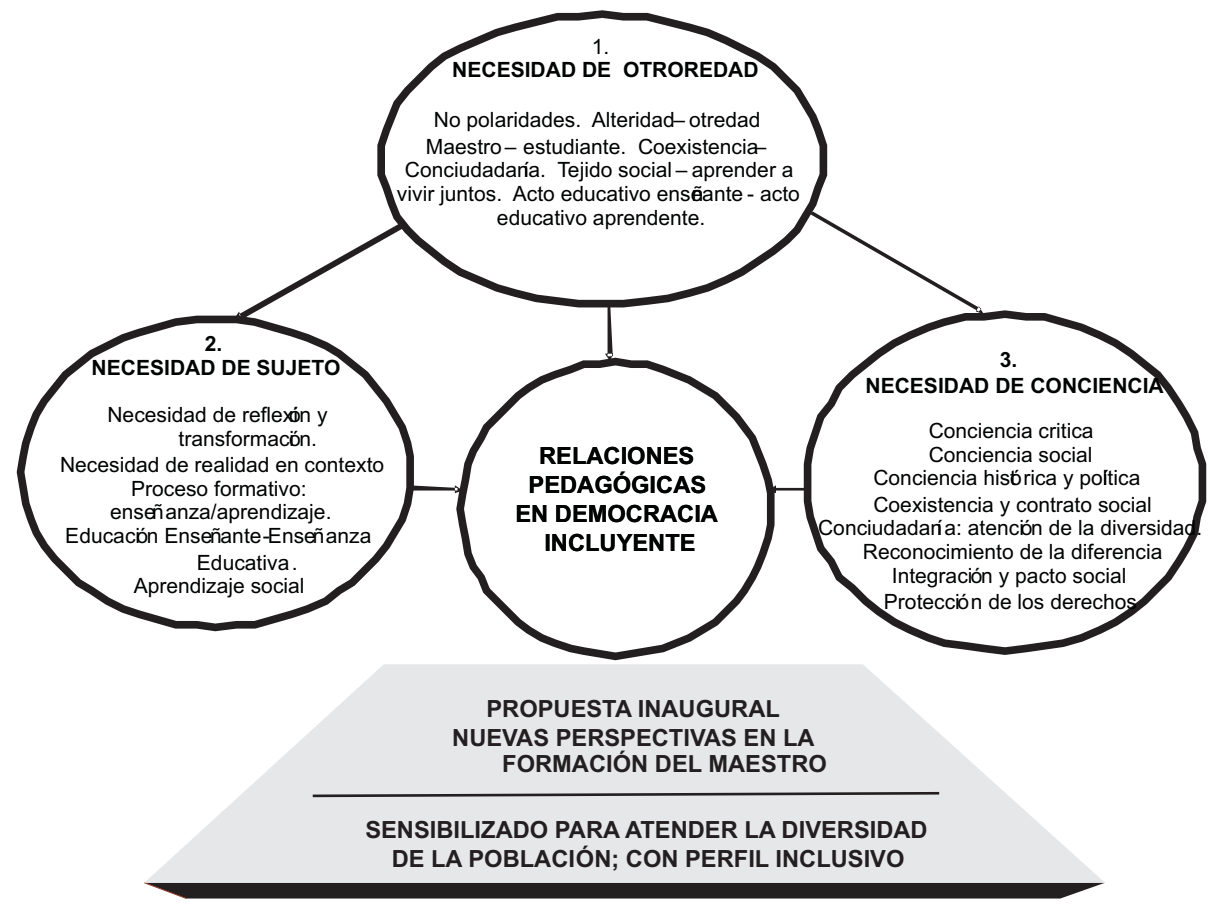

Figura 4. Necesidades emergentes para la construcción de unas Relaciones Pedagógicas en Democracia Incluyente; plataforma de la formación del nuevo/a maestro /a en Colombia.

\section{CONCLUSIONES}

\section{Prácticas Pedagógicas}

* Modulan el desempeño inicial del maestro

* Fortalecen su perfil, son necesarias en la formación

* Potencian los saberes y la experiencia del normalista

* Otorgan autonomía al practicante, le muestran una ruta

* Permiten tomar distancia conceptual de su propia experiencia y de sus maestros/as.

\section{Actores y escenarios}

* Sujetos singulares en ambientes propios de su realidad humana y social. Hacen parte de la diversidad cultural, sus características los ubican dentro de la población vulnerable del municipio de Armenia.

* Los escenarios son también particulares y varían de acuerdo con los sectores. En algunas comunidades, los líderes participan con la organización de las casetas u otros espacios comunales, en otros no hay colaboración, no se percibe 
interés por el cuidado y la protección de los niños y las niñas, y mucho menos por la educación.

\section{Vulnerabilidad Social \\ * Describe las condiciones de marginalidad, pobreza y exclusión, dadas por los efectos de la inequidad, la violencia y el deterioro de la calidad de vida. \\ * Nuevo concepto de estudio y análisis para el normalista superior. \\ *Amplía su campo temático y de acción, confiere liderazgo y actoría social como sujetos públicos de la educación frente a los fenómenos de la complejidad social.}

\section{Formación Inicial del Maestro}

* Directamente asociada con la finalidad de la práctica pedagógica, es una categoría que en la Normal Superior del Quindío se está revisando constantemente, debido al papel que juega este profesional en el desarrollo de la vida humana y en el desarrollo social de un país.

* Proceso para la sensibilización frente a la atención de la diversidad.

*Ampliación del panorama de la escuela.

*Trasciende la esquematización de la enseñanza y el aprendizaje.

*Promueve la descurricularización de los contenidos y las rutinas de la escuela.

*Trasciende las rígidas fronteras de los saberes convencionales.

*Aporta con la creación de un modelo flexible a la política de educación inclusiva en el municipio de Armenia Quindío.

\section{Proceso Incluyente}

*Un acto de innovación que revitaliza la práctica pedagógica inicia.

*Término construido por los actores egresados y practicantes normalistas, quienes lo asimilan con la garantía de derechos de la infancia y la adolescencia, que aporta a la integración, el reconocimiento y la participación social.
*Privilegia en su desarrollo el uso de nuevas metodologías.

*Proyecta el aprendizaje infantil y juvenil y la creación de espacios formativos para la familia y la comunidad.

*Demanda un maestro /a con capacidades y talentos, creativo /a, capaz de relacionarse, brindar afecto y buen trato, que se comprometa con la educación.

\section{Sentido y significado de ser maestro/a}

*Comprensión de sentido.

*Asigna valor especial.

*Entusiasmo y motivación al ser llamados maestros y maestras.

* La experiencia dota de sentido la profesión docente.

* "en esta práctica nos damos a conocer con hechos y no solamente con el discurso, es vivir lo que se hace" * Potencia la actitud pedagógica.

*Propicia construcción y formación permanente del maestro/a.

*Comprensión de la función que el maestro/a cumple en la vida del alumno/a.

* "La experiencia es la que forma".

\section{Carácter de las prácticas pedagógicas}

* Instrumento para construir una sociedad más justa y equitativa.

*Andamiaje que sustenta y retroalimenta la fase de concienciación del sujeto.

* Proyectan la consolidación de un sistema crítico de la enseñanza.

* Potencian la formación del ser humano como miembro activo y participante, comunitaria y socialmente.

*Promueven la creación de otra escuela que agencie la educación inclusiva de calidad, derechos, garantías sociales, diversidad y pluralidad.

*Abren las fronteras de la escuela y de la ciencia.

* Impulsan la modernización y la contemporanización de los currículos (formación docente).

*Conllevan a visionar la oferta de modelos pedagógicos flexibles y currículos alternativos dentro de un marco escolar.

\section{BIBLIOGRAFÍA}

BENNEY y HUGHES (1956) en Taylor S.J y Bogdan R (1987). Introducción a los métodos cualitativos de investigación. Barcelona: Editorial Paidós

BERGER, P L. y LUCKMAN, T. (1.986) La construcción social de la realidad. Buenos Aires: Amorrortu Editores

BERNSTEIN, B (1990). La Estructura del Discurso Pedagógico. Madrid: Ediciones Morata, S. / Mejía Lequerica. 12.

BLANCO y DUK (2000) en UNICEF Y UNESCO (2000) Hacia el desarrollo de las escuelas inclusivas.

BUBER, M en Baeza, H. Martín Buber su vida y obra Revista ALCIONE № 9 (versión electrónica) recuperado de http://www.alcione.cl/nuevo/index.php?object_id=216

CARR, W y KEMIS, S (1986) Teoría Crítica de la Enseñanza. . España: Ediciones Martínez Roca, S.A.

CARR, W (1990) en Rodríguez L. F. (1998) Revista electrónica ínter universitaria de formación del profesorado ISSN 15750965). 
CARR, W (1990) y KEMMIS, (1986) en Rodríguez L. Francisco. (1998) Revista electrónica ínter universitaria de formación del profesorado ISSN 1575-0965.

CHEVALLARD, (1995) en Rodríguez L.F (1998) Revista electrónica ínter universitaria de formación del profesorado ISSN 1575- 0965).

CORAGGIO, J.L (1.994). Pedagogía Crítica, eje de desarrollo de la enseñanza superior. Buenos Aires: Instituto Fronesis.

CZERLOWSKI, M (2.000). Transmisión de la Cultura y la Práctica Pedagógica. Ponencia en Congreso Nacional de Investigación Educativa. Comahue.

DENSE VAILLANT, diciembre 2004, documentos PREAL No. 31.

ELIOT J (1983) en Morín (2001) La mente bien ordenada. Barcelona España: Ed. Seix Barral.

FREINET, C (1960) La educación moral y cívica. Barcelona: Editorial Laia.

FREIRE en Carr W y Kemmis S (1988) Teoría Crítica de la Enseñanza. España: Ediciones Martínez Roca, S.A.

GIMENO (1992) en Rodríguez, L.F (1998) Revista electrónica interuniversitaria de formación del profesorado ISSN 15750965.

GIROUX, H (1987) en Rodríguez L.F(1998) (Revista electrónica ínter universitaria de formación del profesorado ISSN 15750965).

GUARÍN, J G (2004) Caminos / Opciones de Indagación científica: Episteme, Hermenéutica, Conceptos y Métodos. Colombia: Ed. Centro de publicaciones Universidad de Manizales.

HABERMASJ. (1996) La inclusión del otro. Madrid, España: Ediciones Paidos. Ibérica S.A.

KUBIE, Len Maslow, A (1972). La personalidad creadora “Barcelona: Editorial Kairós, S.A.

LATORRE (1992) en Rodríguez L. F (1998) Revista electrónica interuniversitaria de formación del profesorado ISSN 15750965.

MANRIQUE, M. Hacia la inclusión social, tema abordado en el texto producido a partir de la sistematización de la experiencia de las políticas de niñez del Distrito Capital en la administración de Antanas Mockus año 2001-2004.

Ministerio de Educación Nacional (2005) Lineamientos de política para la atención educativa a poblaciones vulnerables.

MORíN, E (2001) La mente bien ordenada. Barcelona España: Edit. Seix Barral.

NOT, L. (1979) Las Pedagogías del Conocimiento. Colombia: Fondo de Cultura Económica.

PÉREZ, G (1992) en Rodríguez L.F (1998) La formación inicial de los maestros en la actualidad: historia de una inconsistencia. Revista electrónica ínter universitaria de formación del profesorado ISSN 1575-0965).

POSNER, G (2001), Análisis del Currículo. Bogotá, Colombia: McGraw-Hill Interamericana, S.A.

ROGERO, J (2000) Conferencia Educación: Igualdad + Diversidad. Valencia, España.

SCHWARTZMAN y STRAUS en Vásquez R. F. (2002) El diario de campo, una herramienta para investigar en preescolar y primaria. Bogotá, D.C: Editorial Enlace editores Ltda.

SHULMAN (1986) en Rodríguez L.F. (1998) Revista electrónica ínter universitaria de formación del profesorado ISSN 15750965.

SILVIA PLATH en Vásquez R. F. (2002) El diario de campo, una herramienta para investigar en preescolar y primaria. Bogotá, D.C: Enlace editores Ltda.

STENHOUSE (1.985) en Rodríguez L.F. (1998) Revista electrónica ínter universitaria de formación del profesorado ISSN 1575-0965).

SUÁREZ P. A. (2000) Cómo acreditar su institución. Bogotá: Orión editores.

VALLS, R (2004) Cuadernos de Pedagogía No. 341.

VAQUERO, G. E. (2.001) Ponencia en Congreso: Construir la escuela desde la diversidad y para la igualdad.

VELASCO, J C, (1998).Introducción al texto La inclusión del otro. Madrid: Instituto de filosofía del C. S. I. C.

WOODS, P en Vásquez R. F. (2002) El diario de campo, una herramienta para investigar en preescolar y primaria. Bogotá, D.C: Editorial Enlace editores Ltda.

ZACCAGNINI, M. Impacto de los paradigmas pedagógicos históricos en las prácticas educativas contemporáneas. Revista Iberoamericana de educación (versión electrónica) Recuperado de www.campus-oei.org.revista.htm.

ZAMBRANO L, A (2007) conferencia "pedagogía, discurso y saber" Colombia.

ZEMELMAN H. (2002) Necesidad de Conciencia. México: Edit. Anthropos.

ZEMELMAN, H (2007) citado en Novalis (2007) recuperado de http://www.mundoprosa.com/foro/archive/index.php/t2536.html 